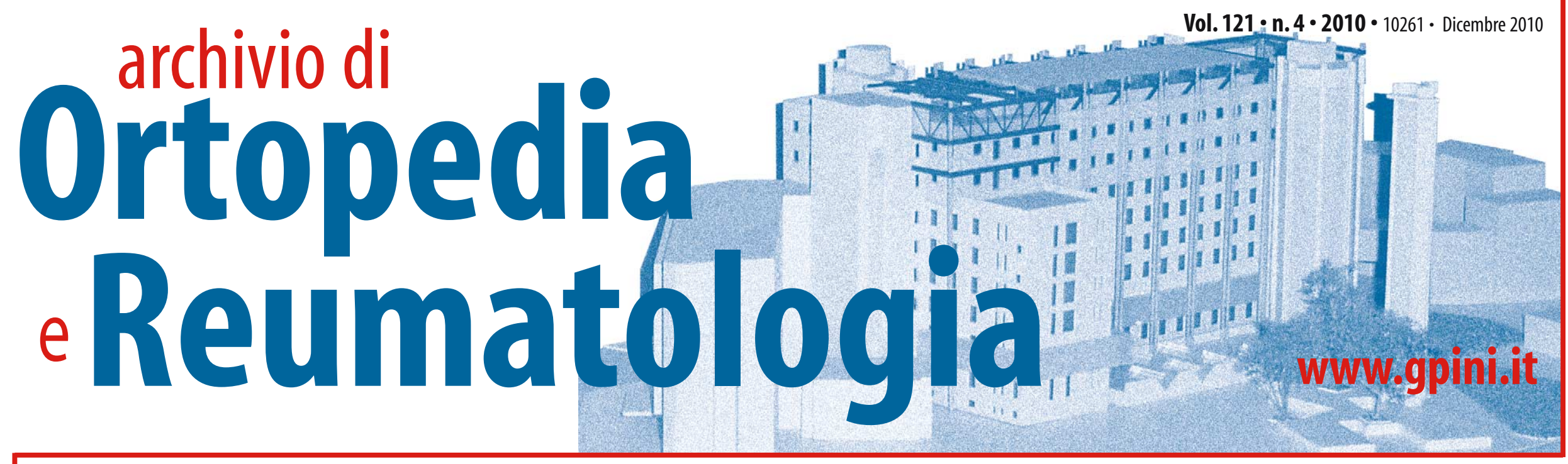

\title{
La caviglia dallo sport alla vita quotidiana. Conferme, aspettative e futuro
}

\author{
a cura di P. F. Bottiglia Amici-Grossi, R. Benvenuti
}

\section{Editoriale}

DOI 10.1007/s10261-010-0046-3

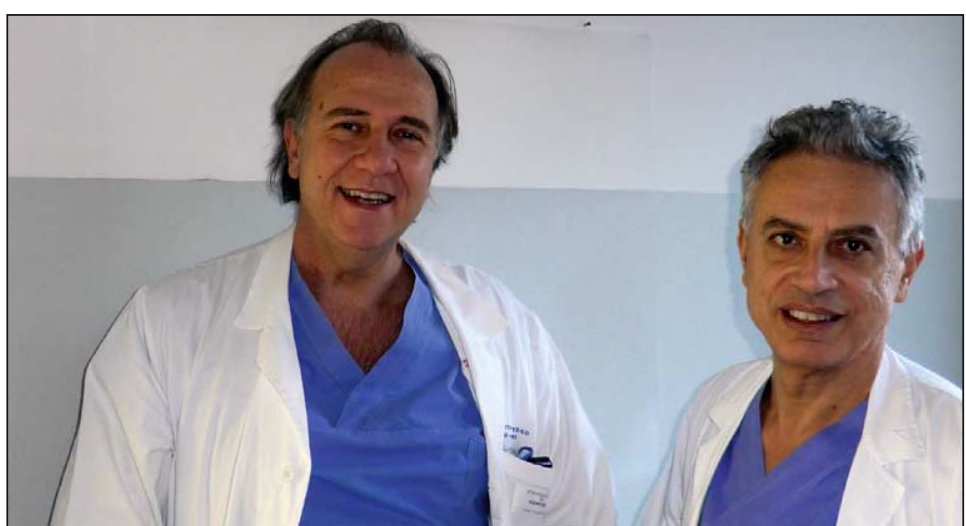

P.F. Bottiglia Amici-Grossi (a sinistra nella foto) e R. Benvenuti

a caviglia e la sua patologia - possono essere coinvolte e interessate naturalmente non soltanto nell'attività sportiva, ma anche nella vita di tutti i giorni

Solo negli Stati Uniti ogni giorno avvengono oltre 30.000 casi di distorsioni di caviglia, che rappresentano il 40 per cento degli infortuni sportivi, ed è quindi naturale immaginare quanta importanza rivesta un corretto approccio diagnostico e terapeutico relativo alle patologie di questa articolazione, dal punto di vista sia sociale sia sanitario.

Il cambiamento radicale della vita di relazione della popolazione nei Paesi evoluti, confermato dal numero crescente anche in età più avanzata di sportivi amatoriali e non, ha portato a un'aumentata attenzione da parte della ricerca e dei clinici per affinare tecniche meno invasive, più precise e selettive, come l'artroscopia, o estremamente radicali come la protesizzazione e il trapianto da cadavere. Le tecniche artroscopiche, soprattutto in ambito sportivo, con la loro continua evoluzione negli anni hanno permesso una sempre maggiore precisione in ambito diagno- lo specifico gesto atletico e del suo meccanismo fisiopatologico.

Non è da dimenticare anche l'importanza dell'imaging tradizionale, della TAC e della RMN, che con le loro complementarizzazioni garantiscono diagnosi e "planning" preoperatori indispensabili ed estremamente precisi. La precisione della diagnosi strumentale, sempre associata all'esperienza del clinico permette quindi la scelta del più corretto trattamento, e in alcuni casi di evitare un'"inflazione" chirurgica.

Le lesioni della cartilagine e del complesso capsulo-legamentoso naturalmente non rimangono soltanto di pertinenza e secondarie all'attività sportiva, ma coinvolgono un grande numero di "uomini di tutti i giorni", che necessitano di trattamenti ad hoc in funzione delle sempre più lunghe e qualitativamente ottimal aspettative di vita.

Anche la patologia degenerativa, quindi, beneficia di nuove tecniche e di innovativi biomateriali che intervengono e si affiancano a tecniche consolidate, complementariz zandosi fra loro.

Come dimenticare, per esem pio, quanta soddisfazione hanno dato negli anni ai vecchi maestri le artrodesi di caviglia nel combattere la guerra contro il dolore nelle grav artrosi, rimanendo ancora oggi una valida soluzione con tecnica sia a cielo aperto sia artroscopica.

Ma va da sé che la ricerca ne tempi moderni ha consentito come già avviene in maniera più collaudata per altre articolazioni, di trattare con la sostituzione protesica le gra vi degenerazioni articolari seppur con indicazioni limi- tate data la complessità biomeccanica della caviglia. Sono stati poi fatti passi avanti, impensabili sino a qualche anno fa, grazie anche all'educazione sociale sulle donazioni e i conseguenti espianti da cadavere, che hanno permesso l'evoluzione delle tecniche di trapianto articolare. Il positivo risultato finale de trattamenti non può prescindere, però, da un corretto trat tamento riabilitativo.

La riabilitazione non è un capitolo a parte, ma occupa uno spazio fondamentale e integrante.

In particolare negli atleti la personalizzazione e l'intensità del programma, in funzione del trattamento effettuato, del gesto atletico specifico e del livello agonistico, riveste un ruolo decisivo per il ritor no il più precoce possibile al l'attività agonistica, soddisfacendo così il concetto "same sport same level", fondamentale in traumatologia dello sport.

Lo scopo di questo numero, quindi, è stato quello di fare il punto sulle conoscenze at tuali e sulle tecniche più avanzate, sperando di aver stimo lato la curiosità culturale dei colleghi già esperti e di altri che, pur non lavorando in centri iperspecialistici, avran- no così la possibilità di avvicinarsi in maniera più completa a queste patologie.

Siamo orgogliosi di avere ospitato in questo numero cultori della materia in rappresentanza di centri di eccellenza italiani e stranieri e il nostro ringraziamento va a loro, sia per la grande qualità dei contributi scientifici, sia per l'entusiasmo e l'amicizia dimostrataci nell'aderire a questa nostra iniziativa.

Un grazie ai colleghi che insieme a noi trattano questi argomenti nel nostro Istituto e hanno condiviso un progetto editoriale basato principalmente su contributi "esterni", nonostante la patologia di caviglia, sia in ambito sportivo sia nella vita di tutti i giorni, rappresenti un momento importante nelle nostre esperienze lavorative quotidiane, scientifiche e chirurgiche.

P. F. Bottiglia Amici-Grossi R. Benvenuti

I Divisione Ortopedia e Traumatologia,

Clinica Ortopedica e Traumatologica,

Centro Studi e Ricerche in Traumatologia dello Sport, Università degli Studi di Milano, Istituto Ortopedico G. Pini, Milano 